\title{
Linear and nonlinear optical properties of borate crystals as calculated from the first principles
}

\author{
DING-SHENG WANG \\ Institute of Physics, Chinese Academy of Sciences, Beijing 100080, China
}

\begin{abstract}
With the development of the state-of-the-art band calculation scheme and massively parallel processing in the high performance computing, we are now able to calculate all important physical properties, including (i) the nonlinear susceptibility; (ii) the multiphoton absorption rate; (iii) the birefringence; and (iv) the energy gap, from the first principles for complex practical nonlinear optical crystals, such as the borate crystal series, with an accuracy acceptable for materials development/design, and answer the questions often raised by the material scientists.
\end{abstract}

Keywords. Optical property; nonlinear optical crystals; first principles calculation.

\section{Introduction}

The development of nonlinear optical (NLO) materials has gained much impetus from their wide application ever since the first observation of optical second harmonic generation (SHG). Among the most widely used SHG crystals, borates have great potential for the highest quality, and in fact, $\beta$-barium metaborate or $\mathrm{BBO}$ $\left(\mathrm{BaB}_{2} \mathrm{O}_{4}\right)$ and lithium triborate or $\mathrm{LBO}\left(\mathrm{LiB}_{3} \mathrm{O}_{5}\right)$ were first developed in 1980's in China, cesium triborate or $\mathrm{CBO}\left(\mathrm{CsB}_{3} \mathrm{O}_{5}\right)$ developed by a Chinese-Japanese collaboration in 1993, and cesium-lithium borate or CLBO $\left(\mathrm{CsLiB}_{6} \mathrm{O}_{10}\right)$ developed independently by Japan and German groups later. They have become an important series in the last 20 years because of their high damage thresholds and large NLO coefficients. Very recently (see Chen et al 1999), even more borate crystals have been developed mostly for the use in the ultra violet range, e.g. there are $\mathrm{SBBO}\left(\mathrm{Sr}_{2} \mathrm{Be}_{2} \mathrm{~B}_{2} \mathrm{O}_{7}\right)$, $\mathrm{BABO} \quad\left(\mathrm{BaAl}_{2} \mathrm{~B}_{2} \mathrm{O}_{7}\right)$, $\mathrm{KABO}\left(\mathrm{K}_{2} \mathrm{Al}_{2} \mathrm{~B}_{2} \mathrm{O}_{7}\right)$ and $\mathrm{BPO}\left(\mathrm{BPO}_{4}\right)$ crystals. The four most important application related criteria of nonlinear optical crystals, i.e. the harmonic generation efficiency, power damage threshold, acceptance angle and transparency window, are known to be determined, respectively, by (i) the nonlinear susceptibility; (ii) the multiphoton absorption rate; (iii) the birefringence; and (iv) the energy gap of the crystals.

In earlier papers, the electronic structure and the linear optical properties of borate crystals were calculated by various cluster and band theoretical methods. Yet due to the complexity, $a b$ initio band calculation and due interpretation of the NLO properties of borate crystals, or of other similar complex crystals, have not yet been achieved until recently. With the development of the state-of-the-art band calculation scheme and massively parallel processing in the high performance computing, we are now able to calculate all these physical properties for complex practical nonlinear optical crystals, which contain usually 30-100 atoms per unit cell and bears low symmetry, from the first principles with an accuracy acceptable for materials development/design. With above important series of borate crystals as examples, it was shown how the computational approach, which was carried out at present by LAPW and CASTEP scheme in the framework of density functional theory, answers the questions which were long in demand by, and proved a challenge to, the experimentalists. This present communication gives a brief review of the investigation carried out in this direction in the last few years.

\section{Energy bands}

The calculated band gap is listed in table 1. Both LAPW and CASTEP methods give a trend of the band gap of these borates in good agreement with the measured ones though their absolute values are all lower than the experimental data as expected in the framework of the local density approximation (LDA), except that calculated data for KABO given by CASTEP bear rather unexpected deviation larger than $3 \mathrm{eV}$. Namely, the band gap given by LAPW is about $1-1.6 \mathrm{eV}$ less, and that given by CASTEP is usually $2.4-3.0 \mathrm{eV}$ less than the measured one. This prominent deviation in the CASTEP calculation suggests that for such very open structures, the use of the usually quite successful pseudo-potentials needs further improvements.

Calculations reveal also that all these borate crystals have qualitatively similar valence band (VB) structure resulting from the $\mathrm{O}$ orbitals of the $\mathrm{B}-\mathrm{O}, \mathrm{Al}-\mathrm{O}$, and $\mathrm{P}-\mathrm{O}$ anionic group. $\mathrm{B}$ and $\mathrm{Al}$ atoms form 3 or 4 coordinate anion groups, viz. $\left(\mathrm{BO}_{3}\right)^{-3}$ and $\left(\mathrm{AlO}_{3}\right)^{-3}$, or $\left(\mathrm{BO}_{4}\right)^{-5}$ and $\left(\mathrm{AlO}_{4}\right)^{-5}$, but $\mathrm{P}$ atom forms only 4 coordinate anion group $\left(\mathrm{PO}_{4}\right)^{-3}$. Strong bonding in these anion groups gives rise 
Table 1. Energy gap (eV) of borate crystals listed in increasing order. For LAPW results see Li et al (1998), for CASTEP results see Lin et al $(2000,2001)$, and for experimental results see references cited therein.

\begin{tabular}{llcccc}
\hline Crystal & Formula & Atoms/cell & Experiment & LAPW & CASTEP \\
\hline BABO & $\mathrm{BaAl}_{2} \mathrm{~B}_{2} \mathrm{O}_{7}$ & 36 & $6 \cdot 19$ & & $3 \cdot 76$ \\
BBO & $\mathrm{BaB}_{2} \mathrm{O}_{4}$ & 42 & $6 \cdot 43$ & $\Gamma-Z: 4 \cdot 85$ & \\
& & & & $\Gamma: 4 \cdot 88$ & \\
KABO & $\mathrm{K}_{2} \mathrm{Al}_{2} \mathrm{~B}_{2} \mathrm{O}_{7}$ & 39 & $6 \cdot 87$ & & $3 \cdot 24$ \\
CLBO & $\mathrm{CsLiB}_{6} \mathrm{O}_{10}$ & 72 & $6 \cdot 87$ & & $4 \cdot 32$ \\
CBO & $\mathrm{CsB}_{3} \mathrm{O}_{5}$ & 36 & $7 \cdot 26-7 \cdot 28$ & $\Gamma: 5 \cdot 86$ & 4.46 \\
LBO & $\mathrm{LiB}_{3} \mathrm{O}_{5}$ & 36 & $7 \cdot 78-7 \cdot 98$ & $\Gamma: 6.95$ & 4.83 \\
BPO & $\mathrm{BPO}_{4}$ & 12 & $8 \cdot 84$ & & $5 \cdot 88$ \\
\hline
\end{tabular}

to a wide separation between occupied $\mathrm{O} 2 p$ orbitals and the empty $\mathrm{B}, \mathrm{Al}$ and $\mathrm{P}$ dominant orbitals. Obviously, a good (wide gap) material could be achieved if a crystal contains only these anion groups by sharing $\mathrm{O}$ ions to satisfy the neutrality, or the cations, which are used to neutralize these anion groups and form stable crystals, do not contribute orbitals within this wide gap. In BPO crystal, the former case happens, and it has the widest gap in this borate series.

In other crystals, there are metallic cations $(\mathrm{Li}, \mathrm{K}, \mathrm{Sr}$, $\mathrm{Cs}$, and $\mathrm{Ba}$ ) which enter into the structure, but they have less strong interaction with $\mathrm{O}$ atoms. Instead, they are dispersed either between partially connected coplanar $\left(\mathrm{B}_{3} \mathrm{O}_{6}\right)^{-3}$ anion groups in $\mathrm{BBO}$ crystal, or between a fully connected skeleton consisting of above $\mathrm{B}-\mathrm{O}, \mathrm{Al}-\mathrm{O}$, and $\mathrm{P}-\mathrm{O}$ anion groups in other crystals. Appearance of these cation orbitals in the conduction bands (CB) bears prominent difference. Orbital of the lightest cation (Li) does not appear at the $\mathrm{CB}$ bottom at all, but the heavier cations (K, Sr, $\mathrm{Cs}, \mathrm{Ba})$ do have their orbitals shown prominently at the $\mathrm{CB}$ bottom below the $\mathrm{B}, \mathrm{Al}$, and $\mathrm{P}$ dominant empty orbitals, as shown in the density of states plot in Lin et al (2001). So, LBO, containing only the lightest $\mathrm{Li}$ cation, also has a gap wider than other crystals in this series which contain heavier cations. Appearance of orbitals of heavier cations at the CB bottom decreases the band gap. As the atomic number of cations increases, their effect becomes even stronger, and crystals containing $\mathrm{Ba}$ atoms $(\mathrm{BABO}$ and $\mathrm{BBO}$ ) have the narrowest gap.

Appearance of these heavy cation orbitals at the $\mathrm{CB}$ bottom (or LUMO range according to the cluster calculation) was well recognized in previous band and cluster calculations. However, it was a debating subject that to what extent the optical properties are influenced by this appearance (see Li et al 1998b).

\section{Linear optical properties}

Calculation of the linear optical properties was carried out within the one-electron picture. The interband optical conductivity tensor reads (atomic units)

$$
\boldsymbol{\sigma}(\omega)=\frac{2 \pi}{\omega \Omega} \sum_{c, v, k}|\langle c, \vec{k}|\vec{e} \cdot \vec{p}| v, \vec{k}\rangle|^{2} \boldsymbol{\delta}\left(E_{c \vec{k}}-E_{v \vec{k}}-\omega\right),
$$

where $\Omega$ is the cell volume, $\omega$ the photon energy, $\vec{e}$ the polarization direction of the photon and $\vec{p}$ the electron momentum operator. The integral over the first Brillouin zone has been replaced by a summation over $\vec{k}$ points. The summation includes the VB states $(v)$, and CB states $(c)$, and the subscripted, $E$, the corresponding band energy. The imaginary part of the complex dielectric function, $\varepsilon_{2}(\omega)$, is evaluated from the optical conductivity, $\sigma(\omega)$, according to $\varepsilon_{2}(\omega)=2 \sigma(\omega) / \omega$ Then the real part of the dielectric function, $\varepsilon_{1}(\omega)$, is obtained by the Kramers-Kronig relation. Thus the static dielectric constant in the long wavelength limitation is given by $\varepsilon_{0}=\varepsilon_{1}(0)$. From the complex dielectric function, the linear refractive index reads as

$$
n(\omega)=\left(\frac{\sqrt{\varepsilon_{1}^{2}(\omega)+\varepsilon_{2}^{2}(\omega)}+\varepsilon_{1}(\omega)}{2}\right)^{1 / 2} .
$$

Since the LDA gives band gap smaller than the experiment, the CB energy has been corrected by considering the self-interaction, and following two papers by Levine and Allan (1991a,b), a scissors operator is added to account for this correction approximately and, at the same time, the momentum matrix elements is corrected by a renormalization procedure.

In LBO crystal which contains only light cation, when the photon energy is larger than the band gap, the absorption involves mainly the $\mathrm{O}$ derived states as the initial states, and the B-O bond derived states as the final states. Because there exist intra-atomic transitions, the absorption coefficient rises rapidly from the onset of the spectra. For crystals containing heavier cations, though these cation orbitals dominate the $\mathrm{CB}$ bottom, they exert less affections on the optical transition than the anionic groups do ( $\mathrm{Li}$ et al 1998b), and as in $\mathrm{CBO}$ and $\mathrm{BBO}$, the onset of spectra is only a slowly increasing step, about $1 \mathrm{eV}$ in width, where the valence electrons transit from 
the $\mathrm{O}$ derived initial states to the cation derived final states. Because this transition is an inter-atomic one, the absorption coefficients are not large. Only when photon energies are more than $1 \mathrm{eV}$ above the band gap, and the final states contain the contribution of the $\mathrm{B}-\mathrm{O}$ bonds, the absorption becomes strong.

The static dielectric constants are listed in table 2. The experimental data are estimated from the refractive indexes at the longest measuring wavelength in the transparent region. The relative errors of the calculated values are less than $15 \%$ with respect to the measured ones. The birefringence index, as defined by the difference of the diffraction indexes along its maximum and minimum optical axis is also listed in table 2, showing a very good agreement with the measured data.

\section{Second order susceptibility}

Microscopical expression of the NLO susceptibility was given in the classical treatise by Bloembergen and coworkers and was well documented, though the NLO properties of practical crystals which exhibit usually rather complex structures have not been at the reach of the first principles elucidation before, and band approaches did not gain essential successes in the study of the NLO properties until recently through the efforts of a few groups. Long after the pioneering work by Aspnes (1972), Ghahramani et al $(1990,1991)$ first reported the derivation of the sum rules which gave general formalism of the SHG coefficients free from divergence at the zero frequency limit for insulators, and developed later an elaborate theory in Sipe and Ghahramani (1993) and

Table 2. Static dielectric constants $\left(\varepsilon_{0}\right)$ and birefringence indexes, $\Delta n$, of borate crystals. LAPW data are given in Li et al (1998b), CASTEP data are given in Lin et al (1999, 2000, 2001), and experimental data are from references cited therein.

\begin{tabular}{|c|c|c|c|c|c|}
\hline & \multirow[b]{2}{*}{ Method } & \multicolumn{3}{|c|}{$\varepsilon_{0}$ along axes } & \multirow[b]{2}{*}{$\Delta n$} \\
\hline & & $a$ or $o$ & $b$ or $e$ & $c$ & \\
\hline \multirow[t]{2}{*}{ BABO } & CASTEP & 2.48 & $2 \cdot 33$ & & 0.050 \\
\hline & Experiment & $2 \cdot 46$ & $2 \cdot 30$ & & 0.053 \\
\hline \multirow[t]{3}{*}{$\mathrm{BBO}$} & LAPW & 2.98 & 2.68 & & 0.089 \\
\hline & CASTEP & $2 \cdot 84$ & $2 \cdot 46$ & & $0 \cdot 116$ \\
\hline & Experiment & 2.74 & $2 \cdot 38$ & & $0 \cdot 113$ \\
\hline \multirow[t]{2}{*}{ KABO } & CASTEP & $2 \cdot 43$ & $2 \cdot 27$ & & 0.052 \\
\hline & Experiment & 2.43 & $2 \cdot 23$ & & $0 \cdot 068$ \\
\hline \multirow[t]{2}{*}{ CLBO } & CASTEP & $2 \cdot 29$ & $2 \cdot 12$ & & $0 \cdot 058$ \\
\hline & Experiment & $2 \cdot 21$ & $2 \cdot 06$ & & $0 \cdot 049$ \\
\hline \multirow[t]{3}{*}{$\mathrm{CBO}$} & LAPW & $2 \cdot 60$ & 2.68 & $2 \cdot 55$ & $0 \cdot 040$ \\
\hline & CASTEP & $2 \cdot 42$ & $2 \cdot 48$ & $2 \cdot 58$ & $0 \cdot 048$ \\
\hline & Experiment & $2 \cdot 31$ & $2 \cdot 40$ & $2 \cdot 49$ & 0.058 \\
\hline \multirow[t]{3}{*}{ LBO } & LAPW & $2 \cdot 66$ & 2.71 & $2 \cdot 82$ & $0 \cdot 048$ \\
\hline & CASTEP & $2 \cdot 49$ & $2 \cdot 53$ & 2.63 & 0.045 \\
\hline & Experiment & $2 \cdot 45$ & 2.53 & $2 \cdot 58$ & 0.041 \\
\hline \multirow[t]{2}{*}{$\mathrm{BPO}$} & CASTEP & $2 \cdot 57$ & 2.56 & & 0.0046 \\
\hline & Experiment & $2 \cdot 56$ & 2.54 & & 0.005 \\
\hline
\end{tabular}

Aversa and Sipe (1995) to derive the analytic expressions for the nonlinear response functions that are automatically free of the unphysically divergent term at zero frequency. Besides, the recent work of Dal Corso et al (1996) gave an alternate formalism based on the timedependent density functional theory. Previous first-principles studies of the SHG have been made only in materials with structures as complicated as $\mathrm{SiC}$ polytypes (Chen et al 1994; Rashkeev et al 1998), where the second order response was found to be dependent on structural changes due to polytypism. However, none of these studies were made on practical SHG crystals, where an interpretation of the underlying physical mechanism is long and urgently in demand.

Recently, calculation of the SHG coefficients of practical NLO crystals was made from the first principles and details of the calculation was given by Duan et al (1999a,b) using LAPW band method and Lin et al (2000) using CASTEP software package. Again, in these calculations, the scissor approximation (Levine et al 1991a,b) was also used to bring about a correction to the conduction band energy and the momentum matrix elements. Table 3 lists the results for zero frequency SHG coefficients. All values listed, including the experimental ones, are brought to the same crystallographic axis system with proper exchange of subscripts and sign of values when citation is made from the literature. Their signs and the order of their absolute values, either of different tensor components of the same crystal, or of different crystals, are all in good agreement within given error bars. The calculated SHG coefficients show nearly quantitative coincidence with the experiments. However, the values as calculated by LAPW method for three crystals are larger than the measured ones for all tensor components by

Table 3. SHG coefficients of borate crystals in unit $10^{-9}$ esu (or $\frac{4 \pi}{3} \times 10^{-1} \mathrm{pm} / \mathrm{V}$ ). LAPW results are given in Duan et al (1999a), CASTEP results are given in Lin et al (1999, 2000, 2001), and experimental data are from references cited therein.

\begin{tabular}{|c|c|c|c|c|}
\hline & $\chi^{(2)}$ & LAPW & CASTEP & Experiment \\
\hline \multirow{3}{*}{$\begin{array}{l}\text { BABO } \\
\text { BBO }\end{array}$} & $d_{11}$ & & 1.79 & \pm 1.79 \\
\hline & $d_{22}$ & $-7 \cdot 11$ & $-3 \cdot 32$ & $\begin{aligned} & \pm 3.82 \\
(\text { also } & \pm 5 \cdot 25)\end{aligned}$ \\
\hline & $d_{31}$ & -0.42 & $-0 \cdot 14$ & \pm 0.27 \\
\hline KABO & $\begin{array}{l}d_{33} \\
d_{11}\end{array}$ & -0.05 & $\begin{array}{l}-0.01 \\
-0.76\end{array}$ & $\begin{array}{l}\sim 0 \\
\pm 1.07\end{array}$ \\
\hline CLBO & $d_{36}$ & & $-1 \cdot 30$ & $\pm 2 \cdot 27$ \\
\hline $\begin{array}{l}\text { CBO } \\
\text { LBO }\end{array}$ & $d_{14}$ & $-3 \cdot 45-2 \cdot 91$ & $-1 \cdot 38$ & $\pm 1.79-2.98$ \\
\hline BPO & $\begin{array}{l}d_{32} \\
d_{33} \\
d_{31}\end{array}$ & $\begin{array}{r}4.05 \\
0.24 \\
-3 \cdot 26\end{array}$ & $\begin{array}{c}1.39 \\
0.033 \\
-1.21\end{array}$ & $\begin{array}{l} \pm 2.03-2.97 \\
\pm 0.15 \\
\pm 1.60-2.75 \\
\pm 1.40-1.86\end{array}$ \\
\hline & $\begin{array}{l}d_{15} \\
d_{14}\end{array}$ & & $\begin{array}{r}-0.85 \\
2.33\end{array}$ & \\
\hline
\end{tabular}


about 1.6 times at the most. On the contrary, the values as calculated by CASTEP method are smaller than the measured ones for all tensor components for all four crystals, by a factor about 0.6 at the extreme. One possible reason for this deviation is possibly the underestimation of the contribution of the near core region to the momentum matrix elements in the pseudo-potential method. Considering the complexity of the crystals under consideration, above consistent agreement should be considered not far from the best possible expectation.

Besides, a scheme of spectral and spatial decomposition is proposed in Duan et al (1999b), and a similar scheme of real-space atom-cutting is proposed in Lin et al (1999) too. They are designed to elucidate the role of each kind of ions and different electron states in such complex crystals. It is shown that, for the main SHG components of these borate crystals, the dominant source of the optical nonlinearity is the nonlinear response of the high-lying occupied $2 p$ electrons of oxygen atoms, while the metallic cations $(\mathrm{Li}, \mathrm{K}, \mathrm{Sr}, \mathrm{Cs}, \mathrm{Ba})$ play minor role. Not only for the lighter $\mathrm{Li}$ and $\mathrm{K}$ cation which contribute negligibly to the SHG coefficient, even in the KABO crystal, when the heaviest $\mathrm{Ba}$ cation states dominate the conduction band minimum by about a few $\mathrm{eV}$ in width, its contribution to the SHG coefficient counts to just $12 \%$, according to Lin et al (2001). This analysis clarified some long existing debates which were raised in previous cluster calculation, mostly due to the uncertainty in the boundary treatments.

Another possible approach in the calculation and analysis of the static second order susceptibility was outlined by Scandolo and Bassani (1995). They proved that in the static limit when the photon energy is far below the transition, the Miller's constant of the second order susceptibility is an average of the third derivative of the crystal potential acting on the electron over the crystal cell, and it depends only on the ground state property of the system. This also explains why the LDA band scheme, which is not appropriate in describing the excitation, gives quite reasonable agreement in its calculation of the susceptibility, at least, when the static limit is of concern.

\section{Multiphoton absorption}

As most important NLO materials especially in the high power applications are inorganic ionic crystals, and in such crystals the multiphoton absorption (MPA) processes are identified as responsible for their intrinsic laser-induced damage (see Jones et al 1989), a clear picture and calculation of MPA coefficients are required. For calculating the MPA coefficients, we use the conventional time-dependent perturbation theory (Li et al 1998a). The electronic transition probability rate per unit volume between the initial VB states and final $\mathrm{CB}$ states by simultaneously absorbing $m$ photons $(m \geq 2)$ from a linear polarized monochromatic light beam can be expressed as

$$
W^{(m)}=2 \pi\left[\frac{2 \pi}{n c \omega^{2}}\right]^{m} \sum_{c, v, \vec{k}}\left|T^{(m)}\right|^{2} \boldsymbol{\delta}\left(E_{c \vec{k}}-E_{v \vec{k}}-m \omega\right),
$$

where $n$ is the refractive index, $c$ the velocity of light in the vacuum, $I$ the incident radiation intensity and $T^{(m)}$ the multiphoton transition amplitude. For given polarization direction $\vec{e}, T^{(m)}$ can be written as

$$
T^{(m)}=\langle c, \vec{k}|M G(m-1) M G(m-2) \ldots M G(1) M| v, \vec{k}\rangle,
$$

where $M=\vec{e} \cdot \vec{p}$ and $G(l)=\sum_{j} \frac{|j, \vec{k}\rangle\langle j, \vec{k}|}{E_{j \vec{k}}-E_{\vec{k}}-l \omega}$, with summation over $j$ covering all the intermediate states. The $m$ photon absorption coefficient $\alpha^{(m)}$, related to the $m$-photon transition probability rate, $W^{(m)}$, is

$$
\boldsymbol{\alpha}^{(m)}=2 m \omega W^{(m)} / I^{m} .
$$

Historically, the analytical calculations of the MPA coefficients usually involved the approximation of the oscillator strengths as well as the energy band, and the choice of the intermediate states. The results of such simple analytical models were believed as generally inaccurate and unreliable. On account of the large discrepancy between the experimental data and these former results, it was concluded that more reliable calculations should be based on accurate first-principles band structures.

However, it is not trivial to calculate the MPA coefficients from the first principles band calculation, because the present tractable first principles band calculation employing the local density approximation which is valid only for the ground state of an inhomogeneous electron system, and is not in concept suitable to excitation problem. It is only from a practical point of view, the LDA has been accepted as a computationally expeditious way to approach this multiphoton problem. We have thus started by verifying the validity of calculation of the

Table 4. Calculated multiphoton absorption coefficients and experimental damage threshold of borate crystals (Li 1997).

\begin{tabular}{ccccc}
\hline & 2-photons $(\mathrm{cm} / \mathrm{GW})$ & 3-photons $\left(10^{-5} \mathrm{~cm}^{3} / \mathrm{GW}^{2}\right)$ & 4-photons $\left(10^{-9} \mathrm{~cm}^{5} / \mathrm{GW}^{3}\right)$ & Threshold $\left(\mathrm{GW} / \mathrm{cm}^{2}\right)$ \\
\hline LBO & $0 \cdot 5 \sim 1$ & $0 \cdot 4 \sim 1$ & $0 \cdot 1 \sim 0 \cdot 5$ & 25 \\
CBO & $0 \cdot 6 \sim 2$ & $0 \cdot 5 \sim 2$ & $0 \cdot 5 \sim 1$ & 26 \\
BBO & $1 \sim 2$ & $1 \sim 3$ & $1 \sim 3$ & 15 \\
\hline
\end{tabular}


MPA coefficients on alkali halides, and other II-VI and III-V ionic crystals, and covalent Ge crystals in Zhang et al (1996), Duan et al (1998), and Li et al (1998a). Comparison with experimental results is quite encouraging.

Though there is no experimental results available for borate crystals yet, calculation was made by Li (1997) and compared with the damage threshold of these crystals (table 4). We do see that LBO and CBO which have damage threshold twice higher than the $\mathrm{BBO}$ crystal have their 2-, 3-, and 4-photon absorption coefficients about half smaller than that of the BBO crystal, in very good agreement with the damage mechanism, though, at present, the quantitative estimation of the threshold is still impossible.

\section{Summary}

In summary, all four main properties of practical nonlinear optical crystals can now be calculated $a b$ initio with accuracy acceptable for experimentalists in their materials development/design. This computational approach also helps a quantitative and instructive understanding and analysis for mechanism and the role played by each kind of ions or chemical bonds in such complex nonlinear optical crystals. It is expected that joining with the molecular dynamic simulation, which has the capability of handling the lattice relaxation effect after atomic substitution, an in-house interactive materials design software could be realized and offers tremendous help to the experimentalists.

\section{Acknowledgements}

This work was supported by the National Pan-deng Project (G19990328-02) of China, and the author is grateful to Professor Chuang-tian Chen for his stimulating discussions and permission of listing their results prior to publication.

\section{References}

Aspnes D E 1972 Phys. Rev. B6 4648

Aversa C and Sipe J E 1995 Phys. Rev. B52 14636

Chen C T, Ye N, Lin J, Jiang J, Zeng W R and Wu B C 1999 Adv. Mater. 111071

Chen J, Levine Z H and Wilkins J W 1994 Phys. Rev. B50 11514

Dal Corso A, Mauri F and Rubio A 1996 Phys. Rev. B53 15638

Duan C G, Gu Z Q, Li J and Wang D S 1998 Phys. Rev. B57 10

Duan C G, Li J, Gu Z Q and Wang D S 1999a Phys. Rev. $\mathbf{B 5 9}$ 369

Duan C G, Li J, Gu Z Q and Wang D S 1999b Phys. Rev. B60 9435

Ghahramani E, Moss D J and Sipe J E 1990 Phys. Rev. Lett. 64 2815

Ghahramani E, Moss D J and Sipe J E 1991 Phys. Rev. B43 8990

Jones S C, Braunlich P, Casper R T, Shen X and Kelly P 1989 Opt. Eng. 281039

Levine Z H and Allan D C 1991a Phys. Rev. Lett. 6641

Levine Z H and Allan D C 1991b Phys. Rev. B44 12781

Li J 1997 Theoretical study of the electronic structure, linear optical properties and multiphoton absorption of alkali halides and borates, $\mathrm{Ph} \mathrm{D}$ dissertation, Institute of Physics, Academia Sinica, Beijing

Li J, Duan C G, Gu Z Q and Wang D S 1998a Phys. Rev. B57 2222

Li J, Duan C G, Gu Z Q and Wang D S 1998b Phys. Rev. B57 6925

Lin J, Lee M H, Liu Z P and Chen C T 1999 Phys. Rev. B60 13380

Lin Z S, Lin J, Wang Z Z and Chen C T 2000 Phys. Rev. $\mathbf{B 6 2}$ 1757

Lin Z S, Wang Z Z, Chen C T and Lee M H 2001 J. Appl. Phys. 905585

Rashkeev S N, Lambrecht W and Segall B 1998 Phys. Rev. B57 9705

Scandolo S and Bassani F 1995 Phys. Rev. B51 6928

Sipe J E and Ghahramani E 1993 Phys. Rev. B48 11705

Zhang W Q, Zhou Y M, Zhong L P, Nie X L and Wang D S 1996 Opt. Commun. 12661 